\title{
Accuracy of a deep convolutional neural network in detection of retinitis pigmentosa on ultrawide-field images
}

\author{
Hiroki Masumoto ${ }^{\text {Corresp., }}{ }^{1}$, Hitoshi Tabuchi ${ }^{1}$, Shunsuke Nakakura ${ }^{1}$, Hideharu Ohsugi ${ }^{1}$, Hiroki Enno ${ }^{2}$, Naofumi \\ Ishitobi $^{1}$, Eiko Ohsugi ${ }^{1}$, Yoshinori Mitamura ${ }^{3}$ \\ ${ }^{1}$ Department of Ophthalmology, Tsukazaki Hospital, Himeji, Japan \\ 2 Rist Inc., Tokyo, Japan \\ 3 Department of Ophthalmology, Insutitute of Biomedical Science, Tokushima University Graduate School, Tokushima, Japan \\ Corresponding Author: Hiroki Masumoto \\ Email address: h.masumoto@tsukazaki-eye.net
}

Evaluating the discrimination ability of a deep convolution neural network for ultrawidefield pseudocolor imaging and ultrawide-field autofluorescence of retinitis pigmentosa. In total, the 373 ultrawide-field pseudocolor and ultrawide-field autofluorescence images (150, retinitis pigmentosa; 223, normal) obtained from the patients who visited the Department of Ophthalmology, Tsukazaki Hospital were used. Training with a convolutional neural network on these learning data objects was conducted. We examined the K-fold cross validation $(k=5)$. The mean area under the curve of the ultrawide-field pseudocolor group group was $0.998(95 \% \mathrm{Cl}=0.9953-1.0)$ and that of the ultrawide-field autofluorescence group was $1.0(95 \% \mathrm{Cl}=0.9994-1.0)$. The sensitivity and specificity of the ultrawide-field pseudocolor group were $99.3 \%(95 \% \mathrm{Cl}=96.3 \%-100.0 \%)$ and $99.1 \%$ $(95 \% \mathrm{Cl}=96.1 \%-99.7 \%)$, and those of the ultrawide-field autofluorescence group were $100 \%(95 \% \mathrm{Cl}=97.6 \%-\%)$ and $99.5 \%$ (95\% Cl $=96.8 \%-99.9 \%)$, respectively. Heatmaps were in accordance with the clinician's observations. Using the proposed deep neural network model, retinitis pigmentosa can be distinguished from healthy eyes with high sensitivity and specificity on ultrawide-field pseudocolor and ultrawide-field autofluorescence images. 
1 Accuracy of a deep convolutional neural network in detection of retinitis pigmentosa on

2 ultrawide-field images

3

4 Hiroki Masumoto, ${ }^{1}$ Hitoshi Tabuchi, ${ }^{1}$ Shunsuke Nakakura, ${ }^{1}$ Hideharu Ohsugi, ${ }^{1}$ Hiroki Enno, ${ }^{2}$

5 Naofumi Ishitobi, ${ }^{1}$ Eiko Ohsugi, ${ }^{1}$ Yoshinori Mitamura ${ }^{3}$

$6 \quad{ }^{1}$ Department of Ophthalmology, Tsukazaki Hospital, Himeji, Japan

$7 \quad{ }^{2}$ Rist Inc., Tokyo, Japan

$8{ }^{3}$ Department of Ophthalmology, Institute of Biomedical Sciences, Tokushima University Graduate

9 School, Tokushima, Japan

10

11 Corresponding author:

12 Hiroki Masumoto, ${ }^{1}$

13 68-1 Aboshi Waku, Himeji City, Hyogo Prefecture 671-1227, Japan;

14 Tel: +81 79-272-8555; Fax: +81 79-272-8550 
15 Email: h.masumoto@tsukazaki-eye.net

16

17 Abstract

18 Evaluating the discrimination ability of a deep convolution neural network for ultrawide-field

19 pseudocolor imaging and ultrawide-field autofluorescence of retinitis pigmentosa. In total, the

20373 ultrawide-field pseudocolor and ultrawide-field autofluorescence images (150, retinitis

21 pigmentosa; 223, normal) obtained from the patients who visited the Department of

Ophthalmology, Tsukazaki Hospital were used. Training with a convolutional neural network on

these learning data objects was conducted. We examined the $\mathrm{K}$-fold cross validation $(\mathrm{k}=5)$. The

mean area under the curve of the ultrawide-field pseudocolor group group was $0.998(95 \% \mathrm{CI}=$

autofluorescence group were 100\% $(95 \% \mathrm{CI}=97.6 \%-\%)$ and $99.5 \%(95 \% \mathrm{CI}=96.8 \%-99.9 \%)$, 
29 respectively. Heatmaps were in accordance with the clinician's observations. Using the proposed

30 deep neural network model, retinitis pigmentosa can be distinguished from healthy eyes with

31 high sensitivity and specificity on ultrawide-field pseudocolor and ultrawide-field

32 autofluorescence images. 
34

35

36

37 mediated inherited retinal dystrophy (Russel et al., 2017) and it was approved by Food and Drug impairments because of retinal photoreceptor cell degradation (rods and cones) (Fahim, Daiger \& Weleber, 2017). Night blindness is a typical clinical feature of the early stage of RP, which is exacerbated by peripheral visual field narrowing and eventually results in loss of central vision (Fahim, Daiger \& Weleber, 2017). RP occurs at a frequency of one case per 3000-7000 persons with no ethnic preference and is the third leading cause of vision loss in Japan, which experienced a notable increase in the number of RP-related cases of blindness from 1988 to 2008(Xu et al., 2006). RP is diagnosed based on the patient's subjective symptoms, fundus findings, visual field examinations, and electroretinogram. There is currently no treatment performed throughout the world for RP that directly acts on the retina (Hartong, Berson \& Dryia, 2006). In 2017, voretigene neparvovec-rzyl demonstrated the efficacy in patients with RPE65- 
48 identified as therapeutic tools for treatment of RP (Yoshida et al., 2014); however, study results

49 have remained inconclusive.

50 There is a concern that exposure to short wavelength light accelerates retinal degeneration, and

51 wearing sunglasses is recommended (Fahim, Daiger \& Weleber, 2017). Additionally, services

52 such as vocational training, walking training, and independent life skill training are provided for

53 reduction in vision-related quality of life (Fahim, Daiger \& Weleber, 2017). Thus, detecting RP

54 at an early stage is important to prevent worsening in visual function or vision-related quality of

55 life. Furthermore, research on treatments such as artificial retina, gene therapy (Fahim, Daiger \&

56 Weleber, 2017), and iPS (Yoshida et al., 2014) has been advanced in recent years.

57 A striking feature of RP is the appearance of lesions throughout the fundus (Fahim, Daiger \&

58 Weleber, 2017). The Optos ${ }^{\circledR}$ camera (Optos Plc, Dunfermline, Scotland) can acquire wide-angle

59 photographs of the fundus that are suitable for diagnosis, because the device can capture images

60 at a $200^{\circ}$ range in a non-mydriatic state with a pupil diameter of $2 \mathrm{~mm}$ (Hu, Liu \& Paulus, 2016).

61 Ultrawide-field pseudocolor (UWPC) imaging of optoscopic images is versatile and can also be 
62 used in diagnosing diabetic retinopathy, vein occlusion, choroidal masses, uveitis, and other

63 similar diseases (Witmer, Parlitsis, Patel \& Kiss, 2013). Black pigment masses are found in the

64 retina in advanced RP. Conversely, it is difficult to diagnose retinitis pigmentosa sine pigmento

65 or early stage RP in UWPC images (Fahim, Daiger \& Weleber, 2017).

66 Furthermore, the Optos camera can capture fundus autofluorescence (FAF) in the same

67 imaging range which is short wavelength. The FAF represents lipofuscin from predominantly the

68

69

obtained by FAF is considered to complement the UWPC image.

retinal pigment epithelium (Mercado \& Louprasong, 2015). Therefore, FAF is useful for RP diagnosis (Oishi et al., 2016; Robson et al., 2003). In RP eyes, annular enhancement findings are observed in the macular region, whereas granular and mottled attenuation regions are observed in the peripheral region (Oishi et al., 2013). Because retinitis pigmentosa sine pigmento, which are poorly changed in UWPC images, have functional photoreceptor cell abnormality, it can be considered that there is a change in the UWAF image as well. That is, the UWAF image Efforts to apply image-based diagnostics using machine learning to improve medical care 
76 efficiency have been reported (Litjens et al, 2017). Among the current machine learning

77 methods, the deep neural network (DNN) has attracted attention for its use in image-based

78 diagnostics because of its relatively high performance, as compared with conventional machine

79 learning methods (Lecun, Bengio \& Hinton, 2015). However, to the best of our knowledge, no

80 study has yet investigated the utility of a DNN model for image-based RP diagnosis. Most

81 recently, several studies regarding the use of a DNN model to assess the UWPC image values for

82 the diagnosis of retinal disorders have been reported by our team. (Ohsugi et al., 2017; Nagasawa

83 et al., 2018; Nagasato et al., 2018)

84 Therefore, the present study aimed to determine whether the DNN in combination with UWPC

85 images is suitable for image-based RP diagnosis. Because it is difficult to diagnose early stage

86 RP using only UWPC images, the classification performance of RP eyes versus normal eyes

87 using DNN with not only UWPC but also UWAF images was examined in this study.

89 Material and methods 
91 The study was approved by the Ethics Committee of Tsukazaki Hospital (Himeji, Japan) (No 171001) and was conducted in accordance with the tenets of the Declaration of Helsinki. An informed consent was obtained from either the subjects of their legal guardians after explanation

94 of the nature and possible consequences of the study. (shown in the supplemental human studies consent file 1) electroretinograms (ERGs) with the recording protocol conforming to the International Society for Clinical Electrophysiology of Vision standards. The ERGs of all the patients with RP were consistent with rod-cone dystrophy. Patients who were diagnosed with RP had the pathognomonic fundus changes such as attenuated retinal vessels, waxy atrophy of optic nerve

101 head, salt and pepper fundus, and bone-spicule pigment clumping. Patients with uveitis or any

102 disease such as Stargard disease, cone dystrophy that could cause RP-like fundus changes were 103 also excluded. Genetic testing was not performed for each case. As of November 19, 2017, the 
104 number of patients with RP registered in the clinical database of the Ophthalmology Department

105 at Tsukazaki Hospital was 226. Seventy-two atypical RP cases such as sector RP and unilateral

106 RP were excluded. Forty-six cases with complications, such as vitreous hemorrhage, stellate

107 vitreous body, intense cataract distorting the fundus image, previous retinal photocoagulation,

108 and concomitant other fundus diseases, were also exclude. Twenty-five cases in which either

109 UWPC and UWAF images were not obtained were excluded. As a result, the remaining number

110 of patients was 83, and the number of images was 150 .

111 The number of patients with normal eyes registered in the database between October 23, 2017

112 and November 19, 2017 was 2,926. Among them, the number of patients that a physician

113 checked again for retinal diseases was 594. Furthermore, after excluding patients who did not

114 have UWPC and UWAF taken at the same time, the remaining number of patients was 167 , and

115 the number of images was 223.

116 Totally, 373 UWPC and 373 UWAF images (150, RP; 223, Normal) from the ophthalmology

117 database of Tsukazaki Hospital were used. For each of the UWPC and UWAF images, a model 
118 was constructed following the process described below.

119 In this study, we examined the $\mathrm{K}$-fold cross validation $(\mathrm{k}=5)$. This method has been reported

120 in detail. (Mosteller \& Tukey, 1968; Kohavi, 1995) All images are divided into K-groups. The

121 right and the left images of the same patients belong to the same group. (K-1) groups are used as

122 training data, and one group is used as validation data. The images of the training data were

123 augmented by adjusting for brightness, gamma correction, histogram equalization, noise

124 addition, and inversion, so that the amount of training data increased by 18 -fold. The deep

125 convolutional neural network (DNN) model was trained with the augmented training data and

126 the validation data and we analyzed the abilities of the deep learning models with the validation

127 data. The training data and the validation data were separated. The process repeated $\mathrm{K}$ times until

128 each of the K groups becomes a validation data set shown in Figure 1.

Deep Learning Model

131 The DNN model called a Visual Geometry Group - 16 (VGG-16) (Simonyan \& Zisserman, 
132 2014) used in the present study is shown in Figure 2. This type of DNN is known to

133 automatically learn local features of images and generate a classification model (Deng et al.,

134 2009; Russakovsky et al., 2015; Lee et al., 2015). All UWPC and UWAF images were resized to

$135256 \times 256$ pixels.

136 VGG-16 comprises five blocks and three fully connected layers. Each block comprises some

137 convolutional layers followed by a max-pooling layer that decreases position sensitivity and

138 improves generic recognition (Scherer, Müller \& Behnke, 2010). The strides of Convolution

139 layers were 1 and the padding of the layers were "same" so the convolution layers only capture

140 the feature of the image, not downsize. The activation function of the layers was ReLU so that

141 we avoid the vanishing gradient problem (Glorot, Bordes \& Bengio, 2011). The strides of max

142 pooling layers were 2 , so the layers compress the information of the image.

143 There are a flatten layer and two fully connected layers after the block 5 . The flatten layer

144 removes spatial information from the extracted feature vectors, and the fully connected layers

145 compress the information from the previous layers and the last fully connected layers with the 
146 activation function, which is softmax, evaluated the probability of each class (in this study 2

147 classes) and classify the target images. Fine tuning was used to increase the learning speed and to

148 achieve high performance even with less data (Agrawal, Girshick \& Malik, 2014). We used

149 parameters from ImageNet: blocks 1 to 4 as default. The weights of convolutional layers and

150 fully connected layers were updated using the optimization momentum stochastic gradient

151 descent algorithm (learning coefficient $=0.0005$, inertial term $=0.9)($ Qian, 1999; Nestrov,

152 1983).

153 The developed prediction model and training were also performed by machine learning with

154 Python Keras (https://keras.io/ja/) using Python TensorFlow (https://www.tensorflow.org/) as a

155 backend. The training and analysis codes are provided as supplemental dataset file 1.

\section{Heatmaps}

Images were created by overlaying heatmaps of the DNN focus site on the corresponding

UWPC and UWAF images. A heatmap of the DNN image focus sites was created and classified 
160 using gradient-weighted class activation mapping (Selvaraju et al., 2017). The target layer is as

161 the third convolution layer in block 3. The ReLU (Glorot, Bordes \& Bengio, 2011) is represented

162 as backprop_modifier. This process was performed using Python Keras-vis

163 (https://raghakot.github.io/keras-vis/).

165 Outcomes

166 Area under the curve (AUC), sensitivity, and specificity were determined for UWPC and

167 UWAF images using the DNN model described above.

168 We created five models and five ROC curves in five times of the process. Images judged to

169 exceed a threshold were defined as positive for RP, and a ROC curve was created.

170 For sensitivity and specificity, the optimal cutoff values, which are the points closest to the

171 point at which both sensitivity and specificity are $100 \%$ in each ROC curve, were used

172 (Akobeng, 2007). Derivation of the ROC curve was performed using Python scikit-learn

173 (http://scikit-learn.org/stable/tutorial/index.html). 
174

175 Statistical Analysis

176 For comparing patient background, age was tested using Student's t-test. Fisher's exact test

177 was performed for comparing the male-female and right-and-left ratios. In all cases, $\mathrm{P}<0.05$

178 was considered statistically significant. These statistical processes were performed using Python

179 Scipy (https://www.scipy.org/) and Python Statsmodels

180 (http://www.statsmodels.org/stable/index.html).

181 For AUC, a 95\% confidential interval was obtained by assuming a normal distribution and

182 using the average and standard deviation of five ROC curves. The $95 \%$ confidence intervals of

183 sensitivity and specificity were calculated assuming a binomial distribution.

184 Derivation of the confidence intervals of AUC, sensitivity and specificity were obtained using

185 Scipy.

186

Results 
188

189

190

191

192

193

194

195

196

197

198

199

Background

The patient background is described in Table 1. There were no significant differences in age, sex, or the ratio between right and left eyes between the normal and RP groups.

Evaluation of model performance

$(95 \%$ CI $=0.999-1.0)$, respectively. The one example of the ROC curves of the UWPC and

UWAF groups is shown in Figure 3. The sensitivity and specificity of the UWPC group were

$99.3 \%(95 \% \mathrm{CI}=96.3 \%-100 \%)$ and $99.1 \%(95 \% \mathrm{CI}=96.1 \%-99.7 \%)$, and those of the UWAF

group were $100 \%(95 \% \mathrm{CI}=97.6 \%-)$ and $99.5 \%(95 \% \mathrm{CI}=96.8 \%-99.9 \%)$, respectively. As for

the sensitivity and specificity, there are no significant difference between the UWPC and UWAF

groups (sensitivity: $\mathrm{P}=.22$, specificity: $\mathrm{P}=.64$ )

When the threshold was set to 0.5 , one RP image was misclassified as Normal for UWAF, and two Normal images were misclassified as RP and one RP image was misclassified as Normal for 
202 UWPC.

203

204

205

206

207 $99.3 \%$, specificity $=99.1 \%$; UWAF: sensitivity $=100 \%$, specificity $=99.5 \%$ ) with both UWPC and UWAF images. In this way, the multilayered DNN model presents the advantage of 
216 constructing an optimum structure for learning and of identifying local features of complex

217 images with subtle individual differences (Deng et al., 2009; Russakovsky et al., 2015; Lee et al., 218 2015).

219 Because UWAF images can sometimes reveal the findings that are not clearly shown on

220 UWPC images, sensitivity was expected to be higher for UWAF images than UWPC images;

221 however, there was no significant difference between them. The reason for this is because both

222 sensitivity and specificity in UWPC images are close to $100 \%$, the results that would exceed the

223 UWPC sensitivity and specificity, which constitutes a significant difference, cannot be obtained.

224 For both UWPC and UWAF images respectively, only one RP image was misclassified as

225 Normal, and these two misclassified images were from different RP cases. Since the

226 misclassified images in the UWAF and UWPC images were derived from different cases, both

227 images should be classified by Neural Network to prevent overlooking.

228 Simultaneously obtaining UWPC and UWAF images at using the Optos camera, which is a

229 non-mydriatic examination, without a significant increase in time is relatively easy. Even in 
230 areas without an ophthalmologist, if the UWPC and UWAF images can be obtained, it is

231 possible to acquire necessary image data to distantly diagnose RP.

232 However, since RP is a very rare disease with a prevalence of less than $0.1 \%$, to identify only

233 one RP patient, at least 3000 healthy persons would have to be examined (Fahim, Daiger \&

234 Weleber, 2017). A scheme in which the ophthalmologist diagnose all acquired images is very

235 difficult. Using the DNN model, early detection and screening of RP can be more efficiently

236 performed.

237 As the first limitation of the present study, we aimed to evaluate the ability of the combination

238 of the DNN model with UWPC or UWAF images to distinguish between normal and RP eyes,

239 rather than distinguish RP from other fundus diseases or coexisting complications. We focus

240 only on the difference between the RP eyes and the normal eyes. The second limitation is that

241 fundus images of patients with vitreous hemorrhage and advanced cataract were excluded from

242 this study. This identification system presumes screening in telemedicine. There are several

243 complications related to RP, thus, it is impossible to distinguish between these entities and RP 
244 from fundus photographs alone (Fahim, Daiger \& Weleber, 2017). Confirmation of diagnosis

245 requires conventional diagnostic techniques performed by an ophthalmologist, such as a

246 mydriatic eye fundus examination, retinal electrography, and Goldmann perimetry (Fahim,

247 Daiger \& Weleber, 2017). In future, it is necessary to accumulate more Optos image data of RP

248 to improve the reliability of the discrimination ability of the proposed model.

249 The lesion of RP is outside of the vessel arcade and the Optos is necessary for the diagnosis of

250 RP. There are no artificial intelligences for diagnose not only for RP but also for other diseases,

251 although our team report several studies about the DNN model for the diagnosis of retinal

252 detachment, glaucoma, diabetic retinopathy, etc. We have to do further experiments in which we

253 classify RP images with non-RP not normal images and construct the DNN model which can

254 diagnose RP regardless of other diseases such as retinal detachment.

256 Conclusion

257 In conclusion, the proposed method was found to be highly sensitive and specific with both 
258 UWPC and UWAF images for the differential diagnosis of RP. It was suggested that our method

259 has a possibility of detecting RP in clinical practice. However, further experiments are required

260 to develop the artificial intelligence for diagnosis of RP.

262 Acknowledgments

263 The authors would like to thank Enago (www.enago.jp) and Cosley Nakaba for the English

264 language review. We wish to thank Masayuki Miki and Shoji Morita (Department of

265 Ophthalmology, Tsukazaki Hospital, Himeji, Japan).

268 Fahim, A. T., Daiger, S. P., \& Weleber, R. G. (2017). Nonsyndromic retinitis pigmentosa

269 overview.

270 Xu, L., Wang, Y., Li, Y., Wang, Y., Cui, T., Li, J., \& Jonas, J. B. (2006). Causes of blindness

271 and visual impairment in urban and rural areas in Beijing: the Beijing Eye Study. 
272 Ophthalmology, 113(7), 1134-e1.

273 Hartong, D. T., Berson, E. L., \& Dryja, T. P. (2006). Retinitis pigmentosa. The Lancet,

274 368(9549), 1795-1809.

275 Russell, S., Bennett, J., Wellman, J. A., Chung, D. C., Yu, Z. F., Tillman, A., ... \& Cross, D.

276 (2017). Efficacy and safety of voretigene neparvovec (AAV2-hRPE65v2) in patients with

277 RPE65-mediated inherited retinal dystrophy: a randomised, controlled, open-label, phase 3 trial.

278 The Lancet, 390(10097), 849-860.

279 Yoshida, T., Ozawa, Y., Suzuki, K., Yuki, K., Ohyama, M., Akamatsu, W., ... \& Okano, H.

280 (2014). The use of induced pluripotent stem cells to reveal pathogenic gene mutations and

281 explore treatments for retinitis pigmentosa. Molecular brain, 7(1), 45.

282 Hu, Z., Liu, Q., \& Paulus, Y. M. (2016). New frontiers in retinal imaging. International Journal

283 of Ophthalmic Research, 2(3), 148-158.

284 Witmer, M. T., Parlitsis, G., Patel, S., \& Kiss, S. (2013). Comparison of ultra-widefield

285 fluorescein angiography with the Heidelberg Spectralis ${ }^{\circledR}$ noncontact ultra-widefield module 
286 versus the Optos ${ }^{\circledR}$ Optomap ${ }^{\circledR}$. Clinical ophthalmology, 7, 389.

287 Mercado, K., \& Louprasong, A. (2015). A clinical guide to fundus autofluorescence. Review of 288 Optometry, 152(9), 70-74.

289 Oishi, A., Oishi, M., Ogino, K., Morooka, S., \& Yoshimura, N. (2016). Wide-field fundus

290 autofluorescence for retinitis pigmentosa and cone/cone-rod dystrophy. In Retinal Degenerative

291 Diseases (pp. 307-313). Springer International Publishing.

292 Robson, A. G., Egan, C., Holder, G. E., Bird, A. C., \& Fitzke, F. W. (2003). Comparing rod and

293 cone function with fundus autofluorescence images in retinitis pigmentosa. In Retinal

294 Degenerations (pp. 41-47). Springer, Boston, MA.

295 Oishi, A., Ogino, K., Makiyama, Y., Nakagawa, S., Kurimoto, M., \& Yoshimura, N. (2013).

296 Wide-field fundus autofluorescence imaging of retinitis pigmentosa. Ophthalmology, 120(9),

$297 \quad 1827-1834$.

298 Litjens, G., Kooi, T., Bejnordi, B. E., Setio, A. A. A., Ciompi, F., Ghafoorian, M., ... \& Sánchez,

299 C. I. (2017). A survey on deep learning in medical image analysis. Medical image analysis, 42, 
$300 \quad 60-88$.

301 LeCun, Y., Bengio, Y., \& Hinton, G. (2015). Deep learning. nature, 521(7553), 436.

302 Ohsugi, H., Tabuchi, H., Enno, H., \& Ishitobi, N. (2017). Accuracy of deep learning, a machine-

303 learning technology, using ultra-wide-field fundus ophthalmoscopy for detecting

304 rhegmatogenous retinal detachment. Scientific reports, 7(1), 9425.

305 Nagasawa T, Tabuchi H, Masumoto H, Enno H, Niki M, Ohsugi H, Mitamura Y. (2018)

306 Accuracy of deep learning, a machine learning technology, using ultra-wide-field fundus

307 ophthalmoscopy for detecting idiopathic macular holes. PeerJ

308 Nagasato, D., Tabuchi, H., Ohsugi, H., Masumoto, H., Enno, H., Ishitobi, T., Sonobe, T.,

309 Kameoka, M., Niki, M., Hayashi, K., Mitaura, Y. (2018) Deep neural network-based method for

310 detecting central retinal vein occlusion using ultrawide-field fundus ophthalmoscopy, Journal of

311 Ophthalmology

312 Mosteller, F., \& Tukey, J. W. (1968). Data analysis, including statistics. Handbook of social

313 psychology, 2, 80-203. 
314 Kohavi, R. (1995, August). A study of cross-validation and bootstrap for accuracy estimation

315 and model selection. In Ijcai (Vol. 14, No. 2, pp. 1137-1145).

316 Simonyan, K., \& Zisserman, A. (2014). Very deep convolutional networks for large-scale image

317 recognition. arXiv preprint arXiv:1409.1556.

318 Deng, J., Dong, W., Socher, R., Li, L. J., Li, K., \& Fei-Fei, L. (2009). Imagenet: A large-scale

319 hierarchical image database. In Computer Vision and Pattern Recognition, 2009. CVPR 2009.

320 IEEE Conference on (pp. 248-255). Ieee.

321 Russakovsky, O., Deng, J., Su, H., Krause, J., Satheesh, S., Ma, S., ... \& Berg, A. C. (2015).

322 Imagenet large scale visual recognition challenge. International Journal of Computer Vision,

323 115(3), 211-252.

324 Lee, C. Y., Xie, S., Gallagher, P., Zhang, Z., \& Tu, Z. (2015, February). Deeply-supervised nets.

325 In Artificial Intelligence and Statistics (pp. 562-570).

326 Scherer, D., Müller, A., \& Behnke, S. (2010). Evaluation of pooling operations in convolutional

327 architectures for object recognition. In Artificial Neural Networks-ICANN 2010 (pp. 92-101). 
328 Springer, Berlin, Heidelberg.

329 Glorot, X., Bordes, A., \& Bengio, Y. (2011). Deep sparse rectifier neural networks. In

330 Proceedings of the fourteenth international conference on artificial intelligence and statistics (pp.

$331 \quad 315-323)$.

332 Agrawal, P., Girshick, R., \& Malik, J. (2014, September). Analyzing the performance of

333 multilayer neural networks for object recognition. In European conference on computer vision

334 (pp. 329-344). Springer, Cham.

335 Qian, N. (1999). On the momentum term in gradient descent learning algorithms. Neural

336 networks, 12(1), 145-151.

337 Nesterov, Y. (1983). A method for unconstrained convex minimization problem with the rate of

338 convergence O (1/k^2). In Doklady AN USSR (Vol. 269, pp. 543-547).

339 Selvaraju, R. R., Cogswell, M., Das, A., Vedantam, R., Parikh, D., \& Batra, D. (2017, October).

340 Grad-CAM: Visual Explanations from Deep Networks via Gradient-Based Localization. In

341 ICCV (pp. 618-626). 
342 Akobeng, A. K. (2007). Understanding diagnostic tests 3: receiver operating characteristic

343 curves. Acta paediatrica, 96(5), 644-647. 


\section{Figure 1}

K-Fold $(K=5)$ cross validation method.

All images are divided into five groups. Four groups are augmented and then used for training the model, and one group is used as a validation data. The process repeated five times until each of the five groups becomes a validation data. The answers of the neural networks for all images are used for calculating the performance of the neural networks.

Split 01

Split 02

Split 05

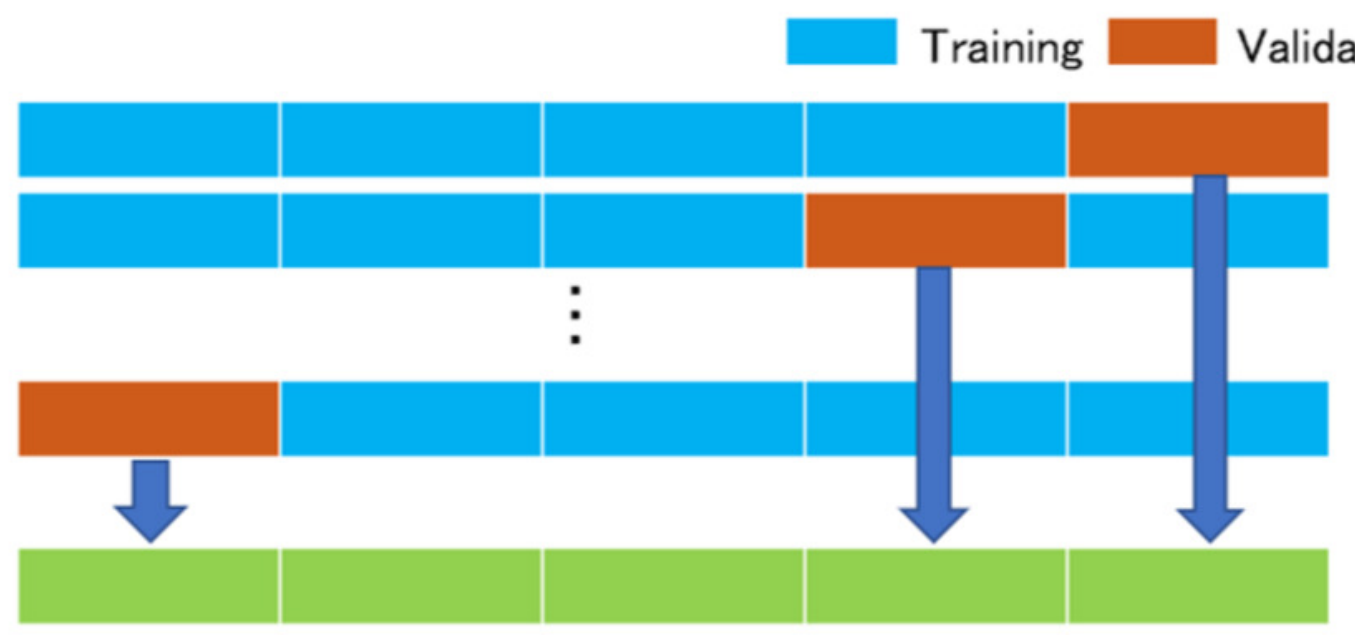




\section{Figure 2}

Overall architecture of the Visual Geometry Group - 16 (VGG-16) model.

VGG-16 comprises five blocks and three fully connected layers. Each block comprises some convolutional layers followed by a max-pooling layer. After flattening the output matrix after block 5 , there are two fully connected layers for binary classification. The DNN used ImageNet parameters as the default weights of blocks 1-4. (Nagasato et al., 2018)

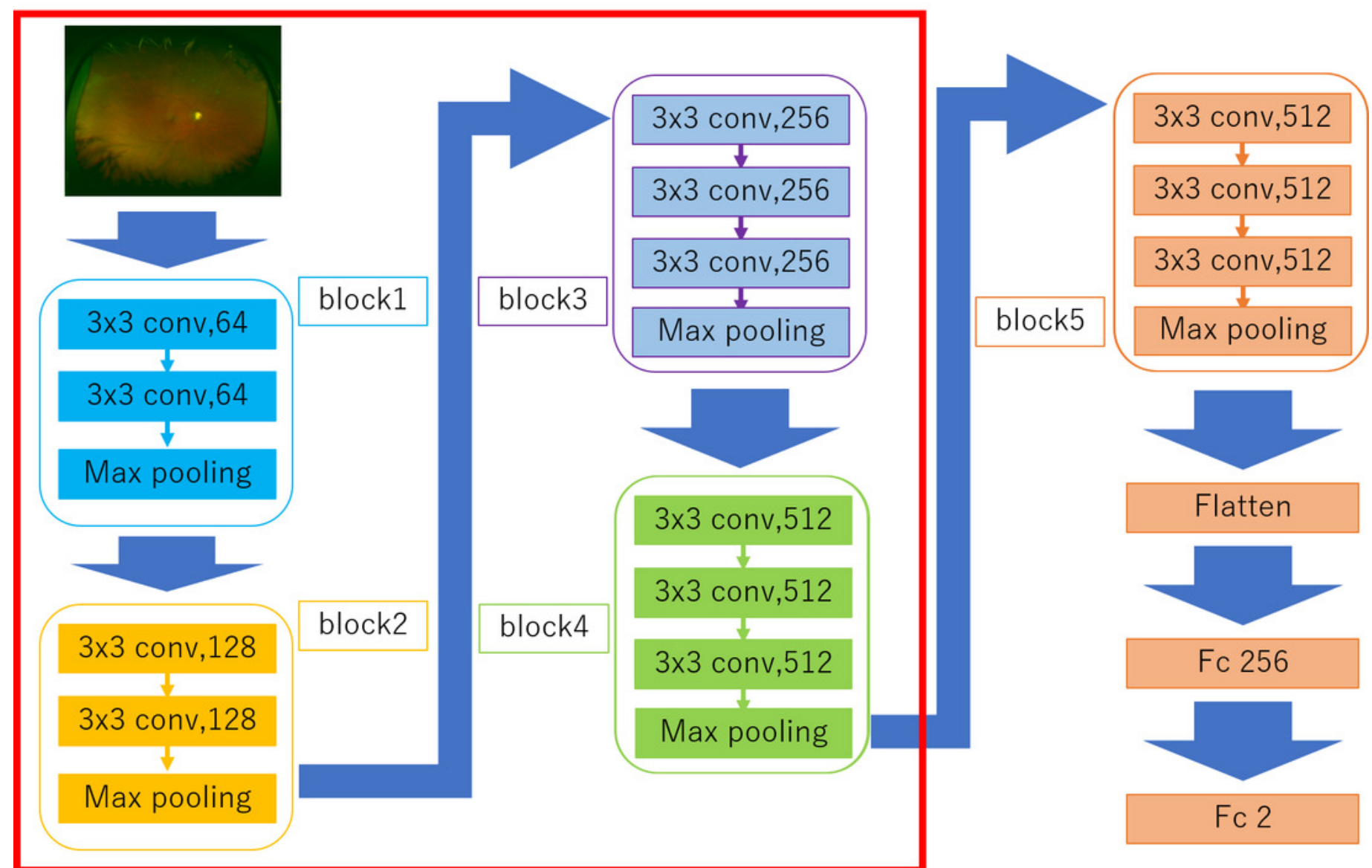


Figure 3

Receiver operating characteristic (ROC) curve of retinitis pigmentosa (RP).

A example of ROC curve of the UWPC and the UWAF.

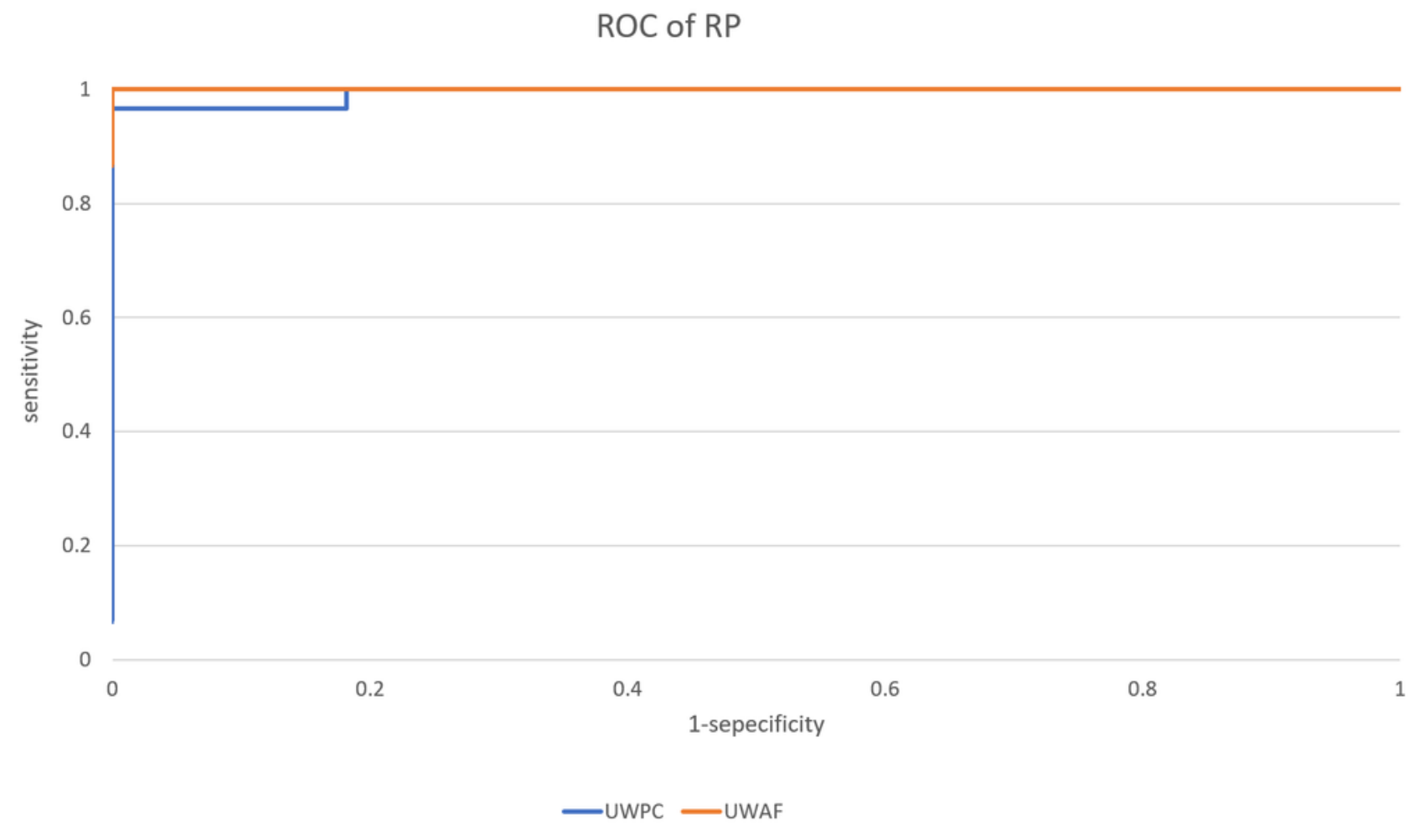




\section{Figure 4}

The images and their heatmaps of (a) ultrawide-field pseudocolor (UWPC). (b) ultrawidefield autofluorescence (UWAF).

In both UWPC and UWAF images, points of interest on the heatmaps accumulates in the bone spicule pigmentation of the fundus, which is characteristic of retinitis pigmentosa.

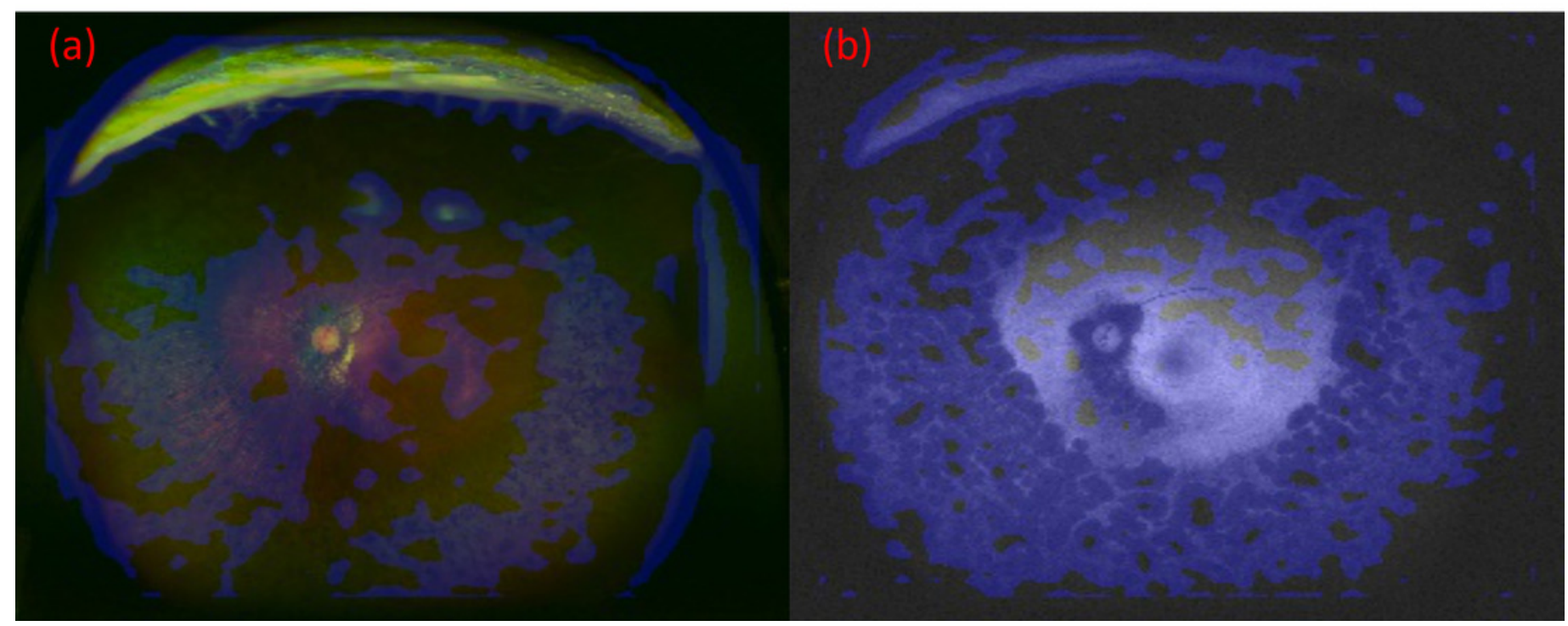




\section{Table 1 (on next page)}

Background characteristics of study participants.

There are no significant differences in age, female ratio and left ratio between normal images and retinitis pigmentosa images 


\begin{tabular}{|c|c|c|c|}
\hline & Normal & $\mathrm{RP}$ & p-value \\
\hline $\mathrm{N}$ & 223 & 150 & \\
\hline Age & $64.0 \pm 14.0(11-78)$ & $61.1 \pm 15.1(19-87)$ & $\mathrm{P}=.06$ (Student's t-test) \\
\hline Sex, female & $123(55.2 \%)$ & $74(49.3 \%)$ & $\mathrm{P}=.29$ (Fisher's exact \\
\hline Eye, left & $119(53.4 \%)$ & $70(46.7 \%)$ & $\mathrm{P}=.21$ (Fisher's exact \\
\hline
\end{tabular}

2 RP: Retinitis Pigmentosa

3 Age (years) is reported as the mean \pm standard deviation with (range).

4 Sex, eye are shown as number with (\%). 